\title{
Breeding biology and novel reproductive behaviour in the Hainan Partridge (Arborophila ardens)
}

\author{
Xiaodong Rao, Canchao Yang ${ }^{*}$ and Wei Liang ${ }^{*}$ (i)
}

\begin{abstract}
Background: The Hainan Partridge (Arborophila ardens) is endemic to Hainan Island, China, and is listed as vulnerable (VU) because its population size is steadily decreasing due to illegal hunting and habitat loss. Its breeding biology is poorly known.

Methods: This study was conducted in three sites in the major tropical primary rainforest in Hainan. Nests of the Hainan Partridge were found by monitoring radio-tracked individuals. Video recorders were set up to monitor reproductive behaviour of Hainan Partridge when nests were found.

Results: Here for the first time we report the breeding biology of Hainan Partridge by providing information on its nest site, nest, eggs, and reproductive behaviour. We found that Hainan Partridges laid immaculate white eggs in ground nests which were totally covered by dry leaves and small branches forming arches that provided optimal concealment. A novel and regular behaviour was found in Hainan Partridges since they grasped leaves and small branches and then threw them on or around their nests to provide cover during incubation and after hatching of chicks.

Conclusions: Recruitment behaviour in Hainan Partridges benefit from supplementation of nest arch material to nests, cover the nest entrance and thus maintain or increase nest concealment. However, it may also contribute to concealment of the track which could expose their activities and clues for predators. Our finding implies that the Hainan Partridge has evolved such a novel reproductive behaviour under the strong selection of predation pressure. Due to habitat fragmentation and habitat loss, we suggest setting up arch structures and provide small branches and leaves in degraded or fragmented habitats to improve their suitability for partridge reproduction. This method may contribute to increasing the reproductive success of Hainan Partridge and thus compensate for its continuous population decrease.
\end{abstract}

Keywords: Habitat fragmentation, Habitat loss, Nest arch, Nest concealment, Recruitment behavior

\section{Background}

There are 21 species of hill partridges (i.e., genus Arborophila) in the family Phasianidae of the order Galliformes (McGowan 1994), and most of them are listed as endangered in the IUCN Red List (IUCN 2017). Generally, these partridge species are poorly studied, especially in terms of behaviour and breeding biology (Madge and

*Correspondence: ccyang@hainnu.edu.cn; liangwei@hainnu.edu.cn Ministry of Education Key Laboratory for Ecology of Tropical Islands, College of Life Sciences, Hainan Normal University, Haikou 571158, China
McGowan 2002). Hainan Partridge (Arborophila ardens) is endemic to Hainan Island, China, and was listed as vulnerable (VU) because its population number is steadily declining due to illegal hunting and habitat loss (Collar et al. 2001; BirdLife International 2010; Liang et al. 2013; IUCN 2017). Recent studies suggested that its current distribution on Hainan Island was its in situ refuge (Chang et al. 2012), and the ancestor of the endemic Hainan Partridge likely originated from Indochina (Chen et al. 2015). However, no information so far has been reported on the breeding biology of Hainan Partridge. 
Here for the first time we report on breeding biology of Hainan Partridges by providing information on its nest site, nest, eggs, and reproductive behavior. We discussed the results and proposed implication for conservation according to its nest characteristics and novel recruitment behavior.

\section{Methods}

\section{Study area and study species}

This study was conducted in Yinggeling National Nature Reserve $\left(18^{\circ} 49^{\prime}-19^{\circ} 08^{\prime} \mathrm{N}, 109^{\circ} 11^{\prime}-109^{\circ} 34^{\prime} \mathrm{E}\right.$; 50,464 ha), Bawangling National Nature Reserve $\left(18^{\circ} 53^{\prime}-19^{\circ} 20^{\prime} \mathrm{N}\right.$, $\left.108^{\circ} 53^{\prime}-109^{\circ} 12^{\prime} \mathrm{E} ; 69,540 \mathrm{ha}\right)$ and Jianfengling National Nature Reserve $\left(18^{\circ} 23^{\prime}-18^{\circ} 52^{\prime} \mathrm{N}, \quad 108^{\circ} 36^{\prime}-109^{\circ} 02^{\prime} \mathrm{E}\right.$; 60,000 ha) on Hainan island, southern China, during the breeding season February-May, 2010-2012. These three study sites constitute the major tropical primary rainforest zone on Hainan island and include the largest continuous tract of this forest type in southern China (Yang et al. 2009, 2011). The Hainan Partridge occurs only in tropical evergreen broadleaf forests and in mixed coniferbroadleaf forests at altitudes of 600-1600 m (Gao 1998). The area of natural forest in Hainan decreased dramatically from $17,000 \mathrm{~km}^{2}$ in the 1940 s to $<3000 \mathrm{~km}^{2}$ in the $1990 \mathrm{~s}$ as a result of overexploitation and unrestricted logging, and expansion of farmlands and oak forest (McGowan et al. 1995). Because of decreases in natural forest area and habitat fragmentation, the population of the Hainan Partridge is decreasing, and the population was estimated at 3900-5200 birds (Baillie et al. 2004).

\section{Radio tracking and nest searching}

We trapped wild Hainan Partridge using custom-built traps which came from the Miao people in China and were modified to capture gamebirds by their legs without harming them (Liang et al. 2003). We outfitted birds with backpack-style transmitters (6.0 g) equipped with activity switches (Holohil Systems Ltd., Canada). We monitored the birds with a radio receiver (TRX-1000S, Wildlife Materials Inc., Murphysboro, IL, USA) and a three-element directional antenna. Nests of Hainan Partridge were found by monitoring the radio-tracked individuals. Video recorders were set up to monitor the reproductive behaviour of Hainan Partridges when nests were found. Egg mass and size were measured using electronic scale (accurate to $0.01 \mathrm{~g}$ ) and vernier calliper (accurate to $0.01 \mathrm{~mm}$ ), respectively, while nest size was measured using a tape (accurate to $0.1 \mathrm{~cm}$ ). Values were given as mean \pm SD. Sample size varied because some nestlings fledged before we found and thus egg size was absent. Furthermore, for some nests that were obviously out of shape due to predation or destruction, nest size was not measured.

\section{Results}

Totally five active nests of Hainan Partridge were found in this study. The nests were built on the ground within a stem base of tree $(n=3)$ or near shrub or liana $(n=2)$, all nests were placed in a bowl lined with dead leaves and feathers and totally covered by dry leaves and small branches forming an arch (Fig. 1; Table 1). Therefore, no nest entrance can be identified from outside the nests because of the cover by leaves and branches and the novel recruitment behaviour of parent birds (see below). The nest and cup diameters were $14.8 \pm 14.0$ and $9.0 \pm 5.7 \mathrm{~cm}$, respectively, with a cup depth of $17.4 \pm 4.0 \mathrm{~cm}(n=3)$ (Table 2). Clutch size was $3.2 \pm 0.8(n=5)$ and the eggs were immaculate white $(13.46 \pm 0.60 \mathrm{~g}, n=4)$ with length and width of $35.82 \pm 1.43$ and $27.62 \pm 0.96 \mathrm{~mm}$, respectively (Table 3 ). Furthermore, a novel and regular behaviour was found in Hainan Partridges when they grasped leaves and small branches and threw them on their nests for cover (Fig. 2; Additional file 1: Video S1) before they left nests, or after they came back to nests, or during incubation. In some cases, they also threw leaves and branches around their nests. Moreover, this behaviour was also observed before the chicks left with parents after hatched (Additional file 2: Video S2). Partridge chicks succeeded to fledge in two out of five nests (Table 1), with a reproductive success of $40 \%(n=5)$. Among them, one nest was destroyed by local people (egg collection), one deserted after being found, and one depredated by an unknown predator (egg lost) (Table 1).

\section{Discussion}

Nests and recruitment behaviour as an adaptation to high risk of predation

Unlike many other Galliforms species (e.g. pheasants) that built exposed ground nests, the nests of Hainan Partridge were situated on the ground with very high concealment. Firstly, small braches and leaves were used to build arches that totally covered the nests. Therefore, the nest entrance was hardly visible and the eggs and birds could not be identified by looking from the outside. This was much different from other reported partridge nests. For example, Sichuan Partridges (Arborophila rufipectus) tend to nest near forest trails and build domed and partially enclosed nests with a 6-8 cm $\times 8-10 \mathrm{~cm}$ entrance (Ji et al. 2007; Fu et al. 2017). In northeastern Thailand, all nests of the Green-legged Partridge (Tropicoperdix chloropus) were placed on the ground between the buttresses of a large tree (DBH range from 27.1 to $109.9 \mathrm{~cm}$ ) in a shallow bowl lined with dead leaves and feathers, and all were on the side of the tree facing downhill away from the nest tree (Ong-in et al. 2016). Secondly and interestingly, the partridges regularly grasped leaves 

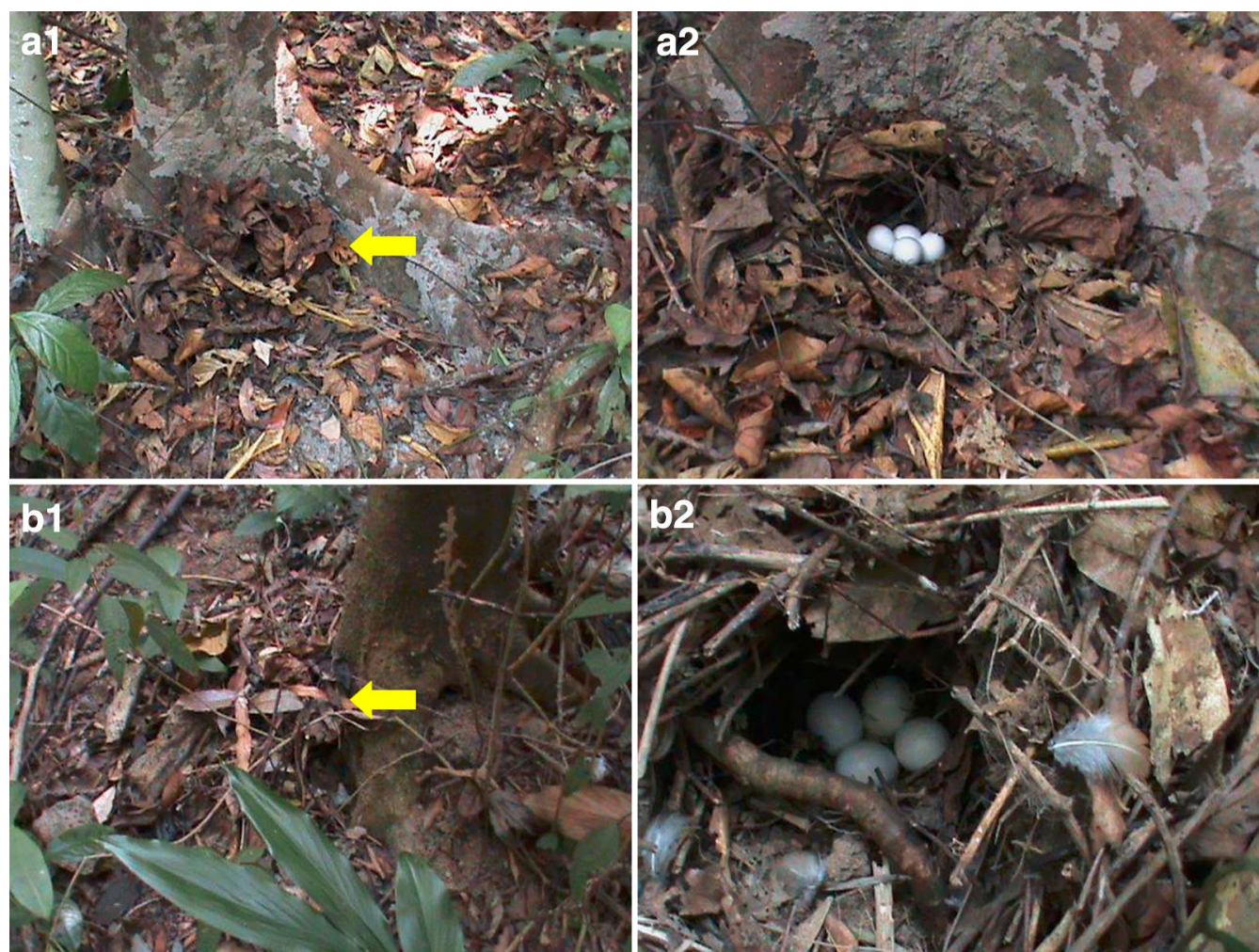

Fig. 1 Nest (a1, b1, indicated by yellow arrows) and eggs (a2, b2) of the Hainan Partridge (A. ardens). a1, b1 refer to the nests whilst $\mathbf{a 2}$, b2 refer to the exposed eggs uncovered by the observer

Table 1 Information on the Hainan Partridge (A. ardens) nests found in this study

\begin{tabular}{|c|c|c|c|c|c|c|}
\hline Nest no. & Clutch size & Nest site & Habitat & Altitude (m) & Study site & Nest fate \\
\hline$\# 1$ & 4 & Stem base of tree & Natural secondary forest & 700 & Yinggeling & Fledged \\
\hline \#2 & 3 & Stem base of tree & Natural secondary forest & 940 & Bawangling & Destroyed \\
\hline \#3 & 4 & Stem base of tree & Natural primary forest & 890 & Bawangling & Depredated \\
\hline \#4 & 2 & Ground near shrub & Natural secondary forest & 1100 & Bawangling & Deserted \\
\hline$\# 5$ & 4 & Ground near liana & Natural primary forest & 300 & Jianfengling & Fledged \\
\hline
\end{tabular}

Table 2 Nest dimensions of the Hainan Partridge ( $A$. ardens)

\begin{tabular}{lccc}
\hline Nest no. & Nest diameter $(\mathbf{c m})$ & Cup diameter $(\mathbf{c m})$ & Cup depth $(\mathbf{c m})$ \\
\hline$\# 1$ & 31.0 & 15.5 & 13.2 \\
$\# 3$ & 6.9 & 5.9 & 21.2 \\
$\# 4$ & 6.5 & 5.5 & 17.9 \\
\hline
\end{tabular}

and small branches and then put them on their nests. To the best of our knowledge, this novel behaviour was never reported for any birds, and may aim to supplement nest arch materials to nests and cover the nest entrance and thus maintain the nest concealment. Additionally, Hainan Partridges also threw leaves and branches around their nests or even before the chicks left after hatching. 
Table 3 Egg mass and size of the Hainan Partridge ( $A$. ardens)

\begin{tabular}{lllll}
\hline Nest no. & Egg no. & Mass $(\mathbf{g})$ & Length $(\mathbf{m m})$ & Width $(\mathbf{m m})$ \\
\hline$\# 1$ & 1 & 13.50 & 32.97 & 28.45 \\
& 2 & 15.80 & 37.15 & 29.20 \\
& 3 & 13.70 & 35.56 & 27.97 \\
$\# 2$ & 4 & 13.80 & 35.50 & 27.82 \\
& 1 & 13.57 & 37.93 & 27.53 \\
& 2 & 13.75 & 38.32 & 28.14 \\
$\# 3$ & 3 & 12.97 & 37.62 & 27.51 \\
& 1 & 13.30 & 34.78 & 28.01 \\
& 2 & 13.90 & 34.84 & 28.02 \\
& 3 & 13.70 & 35.26 & 28.36 \\
\#4 & 4 & 13.00 & 35.51 & 28.22 \\
& 1 & 13.68 & 35.31 & 26.27 \\
& 2 & 11.78 & 34.57 & 26.19 \\
\hline
\end{tabular}

Therefore, this behavior may also contribute to concealment of tracks near nests, which could expose their activities and clues for potential predators. An alternative explanation for such behaviour may be maintenance of heat for eggs, although this needs further investigation. In any case, nest characteristics and novel recruitment behaviour by Hainan Partridges provided optimal concealment to hide their eggs and should be regarded as a specific strategy to reduce nest predation. A recent study using infrared camera recording in the same area (Jianfengling) showed that aerial predators were the main cause of ground nest failure, including Greater Necklaced Laughingthrush (Garrulax pectoralis) and Indochinese Green Magpie (Cissa hypoleuca) (Yang et al. 2016). This may also imply that Hainan Partridges have evolved such a novel reproductive behaviour under strong selection by aerial and hunting predation pressure in local tropical forests (Liang et al. 2013; Wang et al. 2016; Yang et al. 2016).

\section{Implication for conservation}

Under strong selection from predation, Hainan Partridges were only found to live and reproduce in natural forest (Cai et al. 2009; Yang et al. 2009) with dense trees and fallen leaves. However, the area of natural forests in Hainan decreased dramatically from $17,000 \mathrm{~km}^{2}$ in the 1940 s to less than $3000 \mathrm{~km}^{2}$ in the 1990 s as a result of overexploitation and unrestricted logging, and expansion of farmlands and oak forest (McGowan et al. 1995). Compared with degraded or fragmented habitats, natural forest may provide suitable cavity structure, numerous dry leaves and branches for nest building. According to our results, we propose the following suggestions for conservation of the Hainan Partridge. Because Hainan Partridges build nests with arches made of small branches and leaves, provisioning of supplemental nest materials or setting man-made arches at the sites preferred by partridges may improve their suitability as nest sites for nest building and subsequently benefit their reproduction. This could potentially contribute to increasing the reproductive success of Hainan Partridges.

\section{Conclusions}

Here for the first time we provided information on breeding biology of the Hainan Partridge by reporting its nest site, nest, eggs, and reproductive behaviour. A novel and regular behaviour was found in Hainan Partridges that grasped leaves and small branches followed by throwing them on or around their nests for cover during incubation and after hatching. Such behaviour may aim to supplement nest arch material to their nests and cover the nest entrance and thus maintain or increase nest concealment, and also contribute to concealment of the track which could expose their activities and clues for predators. Our finding implies that Hainan Partridge has evolved such novel reproductive behaviour under the strong selection of predation. Due to habitat fragmentation and habitat loss, we suggest that setting up 


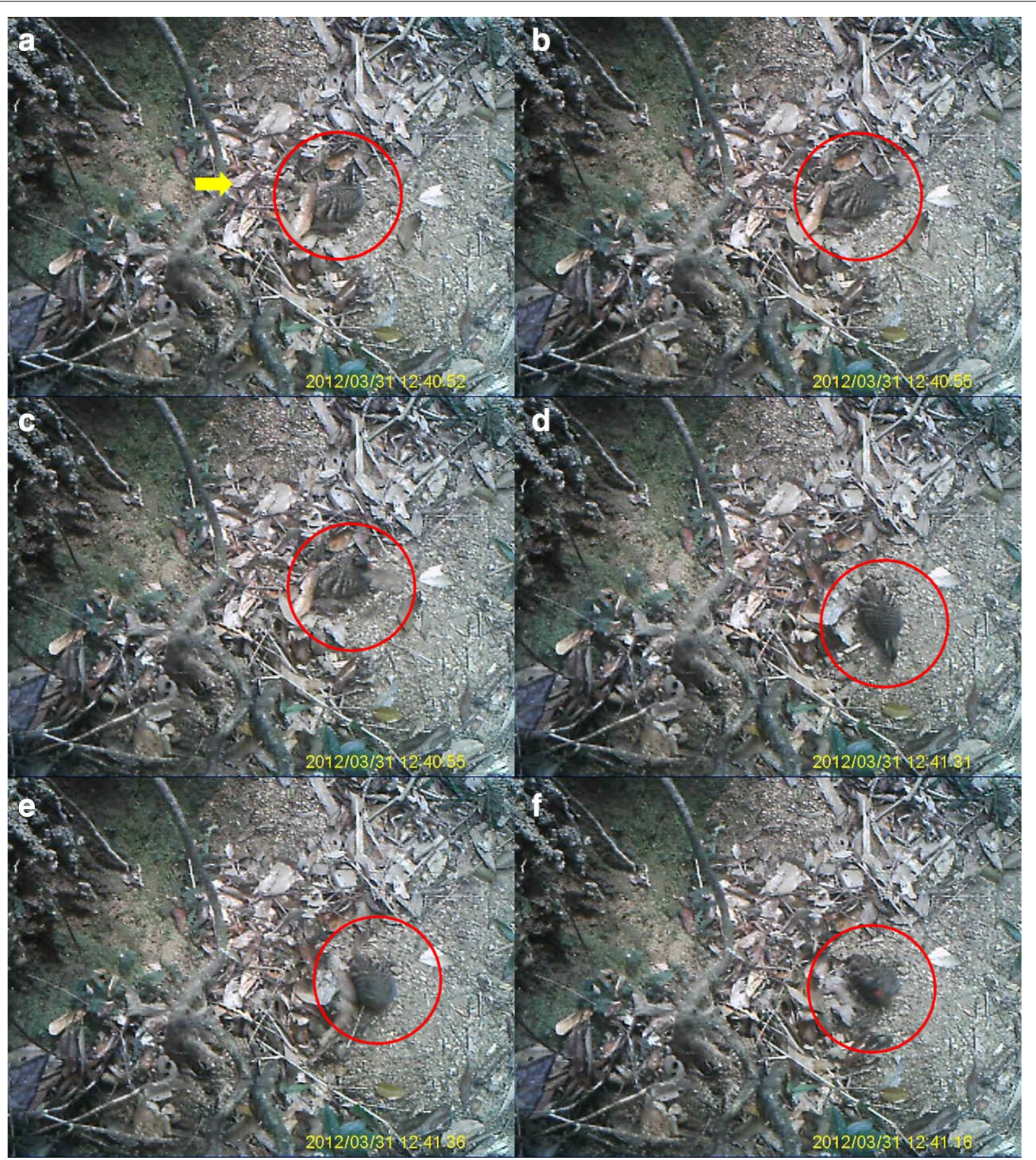

Fig. 2 A Hainan Partridge (A. ardens) grasping leaves and putting them onto its nest (for more details see Additional file 1: Video S1). Letter sequence refers to the time sequence, yellow arrow and red circles indicate the location of nest and partridge, respectively

arch structures and provide small branches and leaves in degraded or fragmented habitats may improve their suitability for partridge reproduction. This method may contribute to increasing reproductive success of Hainan Partridge and thus compensate for its continuous population decline.

\section{Additional files}

Additional file 1: Video S1. A Hainan Partridge was grasping leaves and putting them onto its nest.

Additional file 2: Video S2. A Hainan Partridge chick was throwing leaves and branches around its nest.

\section{Authors' contributions}

WL was responsible for directing field data collection, collaborating with international donors, managing all associated logistical details and permit requirements in China. XR and CY were responsible for data analyses and writing the manuscript, as well as assisting with conducting the research. All authors read and approved the final manuscript.

\section{Acknowledgements}

We thank Prof. Anders P. Møller for kindly helping improve the English text, and Zhongliang Jiang, Jiajia Wang, Junqiu Wu and Xi Cheng for their assistance with fieldwork. We would also thank the Forestry Department of Hainan Province, and Bawangling, Jianfengling and Yinggeling National Nature Reserves, Hainan, China, for kindly permitting us to conduct this study.

\section{Competing interests}

The authors declare that they have no competing interests. 


\section{Availability of data and materials}

The datasets used in the present study are available from the corresponding author on reasonable request.

\section{Consent for publication}

Not applicable.

\section{Ethics approval and consent to participate}

The experiments comply with the current laws of China, where they were performed. Fieldwork was carried out with permission from the Forestry Department of Hainan Province, and Bawangling, Jianfengling and Yinggeling National Nature Reserves, Hainan, China. Experimental procedures were in agreement with the Animal Research Ethics Committee of Hainan Provincial Education Centre for Ecology and Environment, Hainan Normal University (Permit No. HNECEE-2011-006).

\section{Funding}

This work was supported by the National Natural Science Foundation of China (Nos. 30360015 and 30860044 to WL), National Geographic Society (NGS) Conservation Trust (C66-05 to WL) and Program for New Century Excellent Talents in University (NCET-13-0761 to CY).

Received: 7 June 2017 Accepted: 1 December 2017

Published online: 19 December 2017

\section{References}

Baillie JEM, Hilton-Taylor C, Stuart SN. IUCN red list of threatened species: a global species assessment. Gland: IUCN Survival Commission; 2004.

BirdLife International. Species factsheet: Arborophila ardens. http://www. birdlife.org (2010).

Cai Y, Yang C, Liang W. Negative effects of plantations on bird diversity in Yinggeling Nature Reserve, Hainan Island. Sichuan J Zool. 2009;28:764-7 (in Chinese)

Chang J, Chen D, Ye X, Li S-H, Liang W, Zhang Z, Li M. Coupling genetic and species distribution models to examine the response of the Hainan partridge (Arborophila ardens) to late Quaternary climate. PLoS ONE. 2012;7:e50286.

Chen D, Chang J, Li S-H, Liu Y, Liang W, Zhou F, Yao C-T, Zhang Z. Was the exposed continental shelf a long-distance colonization route in the ice age? The southeast Asia origin of Hainan and Taiwan partridges. Mol Phylogenet Evol. 2015;83:167-73.
Collar NJ, Andreev AV, Chan S, Crosby MJ, Subramanya S, Tobias JA. Threatened birds of Asia: the BirdLife International Red Data Book (Part A); 2001.

Fu Y, Dai B, Wen L, Chen B, Dowell S, Zhang Z. Unusual incubation behavior and embryonic tolerance of hypothermia in the Sichuan Partridge (Arborophila rufipectus). J Ornithol. 2017;158:707-15.

Gao Y. Conservation status of endemic Galliformes on Hainan Island, China. Bird Conserv Int. 1998;1998(8):411-6.

IUCN. IUCN Red list of threatened species. Version 2017-1. www.iucnredlist.org (2017). Accessed on 07 June 2017.

$\mathrm{Ji}$ T, He Q, Jiang Y, Li J, Feng S, Ling Z. A short note on the nest of the Sichuan Partridge at Laojunshan Nature Reserve, Sichuan, China. Chin J Zool. 2007;42:88 (in Chinese).

Liang W, Cai Y, Yang C. Extreme levels of hunting of birds in a remote village of Hainan Island, China. Bird Conserv Int. 2013;23:45-52.

Liang W, Zheng G, Zhang Z, Ding C. Habitat use by golden pheasants (Chrysolophus pictus) based on radio-tracking locations. Acta Zool Sin. 2003;2003(49):179-84 (in Chinese).

Madge S, McGowan P. Pheasants, partridges and grouse - a guide to the pheasants, partridge, quails, grouse, guineafowl, buttonquails and sandgrouse of the world. Helm identification guides. London: Christopher Helm; 2002

McGowan PJK. Family Phasianidae. In: del Hoyo J, Elliott A, Sargatal J, editors. Handbook of the birds of the world, vol. 2. Barcelona: Lynx Edicions; 1994.

McGowan PJK, Dowell JP, Carroll JP, Aebischer NJ. Status survey and conservation action plan 1995-1999: partridge, quails, francolins, snowcocks and guineafowl. Reading: IUCN and World Pheasant Association; 1995.

Ong-in T, Pierce AJ, Gale GA, Browne SJ, Tommaso Savini T. Nesting ecology and nest site selection of green-legged partridge. Raffles B Zool. 2016:64:89-97.

Wang J, Yang C, Shi H, Liang W. Reflectance and artificial nest experiments of reptile and bird eggs imply an adaptation of bird eggs against ultraviolet. Ecol Res. 2016;31:105-10.

Yang C, Cai Y, Liang W. Bird diversity in rainy season in forest regions of Hainan Island, China. Chin J Zool. 2009;44:108-14 (in Chinese).

Yang C, Wang J, Liang W. Blocking of ultraviolet reflectance on bird eggs reduces nest predation by aerial predators. J Ornithol. 2016;157:43-7.

Yang C, Zhang Y, Cai Y, Stokke BG, Liang W. Female crowing and differential responses to simulated conspecific intrusion in male and female Hainan partridge (Arborophila ardens). Zool Sci. 2011;28:249-53.

\section{Submit your next manuscript to BioMed Central and we will help you at every step:}

- We accept pre-submission inquiries

- Our selector tool helps you to find the most relevant journal

- We provide round the clock customer support

- Convenient online submission

- Thorough peer review

- Inclusion in PubMed and all major indexing services

- Maximum visibility for your research

Submit your manuscript at www.biomedcentral.com/submit
BioMed Central 